\title{
In vitro Assessment of Polysaccharides Immunoregulatory Effects on CD3+CD56+ NKT-like Cells
}

\author{
Minghao Lu ${ }^{1}$, Yaobiao Huang ${ }^{1}$, Lianyang Yang ${ }^{1,2}$ and Chun Chen ${ }^{1 *}$ \\ ${ }^{1}$ China Jiliang University, Zhejiang Provincial Key Laboratory of Biometrology and Inspection \\ and Quarantine, Hangzhou, 310018, China. \\ ${ }^{2}$ Hangzhou Changhua Biotech, Hangzhou 310018, China.
}

\begin{abstract}
Author's contribution
This work was carried out in collaboration among all authors. Authors ML and YH contributed equally to this study. Author CC conceived and designed the study. Authors ML and LY collected the data. Author CC performed the analysis. Authors CC and ML wrote the manuscript and performed the experiments. All of the authors have read and approved the final manuscript.
\end{abstract}

Article Information

DOI: $10.9734 / A R R B / 2021 / v 36 i 430365$ Editor(s):

(1) Dr. Gunanidhi Dhangadamajhi, North Orissa University, India.

Reviewers:

(1) Tawut Rudtanatip, Khon Kaen University, Thailand. (2) Nditange Shigwedha, University of Namibia, Namibia. Complete Peer review History: http://www.sdiarticle4.com/review-history/67907

\section{ABSTRACT}

Aims: Polysaccharides have shown efficacy in a broad range of applications, especially in immune regulation. However, there are only few effective methods available to evaluate and compare the immunoregulatory properties of the different polysaccharides in vitro. To screen stringently and extend the fields of application of polysaccharide-based treatments, a novel in vitro method, based on the use of cord blood-derived CD3+CD56+ NKT-like cells, was developed in this study.

Methodology: Four injectable polysaccharides used in clinics, including Lentinan (LNT), Bacillus Calmette-Guerin polysaccharide nucleic acids (BCG-PSN), ginseng polysaccharides (GPS), and Shengfu polysaccharides (SFPS), which all might benefit cancer therapy, were further validated by testing their effect on cell proliferation and cytotoxicity in vitro. This new evaluation system could discriminate the effects of the four polysaccharides.

Results: Cells treated for 4 days with either of the four polysaccharides were fewer than untreated cells. Upon 7-day treatments, plant- or bacteria-derived BCG-PSN, GPS, and SFPS induced higher 
level of IFN- $\square$, proportion of cells with a CD3+CD56+ phenotype, and cytolytic activity toward A549 cells, compared to controls. However, fungus-derived LNT yielded lower IFN- $\square$, proportion of CD3+CD56+ cells, and cytolytic activity than control conditions. Additionally, all four polysaccharides, in decreasing order GPS, SFPS, BCG-PSN, and LNT, induced significantly higher concentrations of TNF- $\alpha$ compared to control, with respectively.

Conclusion: Cord blood-derived CD3+CD56+ NKT-like cells may represent a powerful tool to screen and evaluate the immunoregulatory potential of biological or chemical compounds for clinical developments and applications.

Keywords: Polysaccharides; NKT-like cells; immune regulation.

\section{ABBREVIATIONS}

\begin{tabular}{|c|c|}
\hline $\begin{array}{l}\text { ANOVA } \\
\text { BCG-PSN }\end{array}$ & $\begin{array}{l}\text { : Analysis of variance } \\
\text { : Bacillus Calmette-Guerin } \\
\text { Polysaccharide nucleic acid }\end{array}$ \\
\hline GPS & : Ginseng polysaccharide \\
\hline HUCBMNC & $\begin{array}{l}\text { : Human cord blood mononuclear } \\
\text { Cell }\end{array}$ \\
\hline$I F N-\gamma$ & $:$ Interferon- $\gamma$ \\
\hline IL & : Interleukin \\
\hline LNT & : Ientinan \\
\hline$\angle S D$ & : Least significance difference \\
\hline$M H C$ & : Major histocompatibility complex \\
\hline NC & : normal control \\
\hline NK & Natural killer cell \\
\hline NKT-like cell & : Natural killer T-like cell \\
\hline & : Standard deviation \\
\hline SFPS & : Shengfu polysaccharide \\
\hline
\end{tabular}

\section{INTRODUCTION}

Polysaccharides have demonstrated complex biological activities and a variety of functions, especially in immunoregulation $[1,2]$. Potential functions of polysaccharides include immunity enhancement [3], inhibition of bladder cancer cells [4], and alleviation of inflammation and ulcers in the digestive tract [5]. In addition, polysaccharides can promote the production of cytokines, thereby triggering cellular and molecular mechanisms of immune regulation [6]. Many polysaccharide treatments derived from fungi, plants, and bacteria, such as Lentinan (LNT) [7], Bacillus Calmette-Guerin polysaccharide nucleic acids (BCG-PSN) [8], ginseng polysaccharides (GPS) [9], and Shengfu polysaccharides (SFPS) [10], are already used in treatment for immunity-related diseases. However, few in vitro methods, especially using $\mathrm{T}$ cells, have been developed to preevaluate the immunoregulatory effectiveness of polysaccharides before clinical trials.
CD3+CD56+ NKT-like cells are a broad group of CD3+ $T$ cells co-expressing $T$-cell antigen receptor (TCR) and Natural Killer (NK-cell) markers [11,12]. Different from classic $T$ cells, CD3+CD56+ NKT-like cells possess NK and T cell characteristics and have both innate immune function and adaptive immune function [13]. Their dual function aligns CD3+ CD56+ NKT-like cells with NKT cells as first-line innate immune effectors and potential modulators of adaptive immune responses against microorganisms. NKT cells can recognize lipid or glycolipid antigens presented by the MHC class I-related protein CD1d, and carry out multiple functions, including antibacterial and antiviral responses, tumor-related immunosurveillance and immunosuppression, and inhibition or promotion of the development of autoimmune diseases [14].

Several experimental models were proved that the relationship on the immunoregulatory potential of NKT cells against different infectious agents and tumors [15]. However, the physiological function of NKT cells and their possible role in disease pathogenesis remain largely unexplored. To our knowledge, innate CD3+ CD56+ NKT-like cells account for about $5 \%$ to $15 \%$ of the peripheral T cell, and about $50 \%$ of $\mathrm{T}$ cells in the liver [16]. Furthermore, the activation of CD3+CD56+ NKT-like cells may be critical because they are an early source of regulatory cytokines, such as tumor necrosis factor-alpha (TNF- $\alpha$ ) and interferon-gamma (IFNY), have immediate killing functions associated with degranulation [17]. Previous studies have already proved that CD3+CD56+ NKT-like cells are absent from the cord blood [18]. Therefore, a novel evaluation method based on cord bloodderived CD3+CD56+ NKT-like cells was developed for in vitro evaluation of four common polysaccharide treatments which might benefit cancer therapy in this study. 


\section{MATERIALS AND METHODS}

\subsection{Induction of CD3+CD56+ NKT-like Cells}

Human umbilical cord blood mononuclear cells (HUCBMNC) from healthy donors who had signed a consent form were prepared by FicollHypaque density gradient centrifugation (Amersham Biosciences, Canada). HUCBMNCs were cultured in Stem Cell Growth Medium (SCGM, CellGenix Technologie Transfer GmbH) supplemented with 10\% AB-human serum (PANBiotech, Germany) and $1000 \mathrm{U} / \mathrm{mL}$ of IL-2 (Shanghai Huaxin Biotechnology Co., Ltd., China) for $24 \mathrm{~h}$ in 12-well plates. Mouse antihuman CD3 monoclonal antibody (YES Biotechnology Co., Ltd., Canada), was added at $100 \mathrm{ng} / \mathrm{mL}$ and incubated at $37^{\circ} \mathrm{C}$ and $5 \%$ $\mathrm{CO}_{2}$.

\subsection{Proliferation of CD3+CD56+ NKT-like Cells Co-cultures with four Poly- saccharide Injections}

The above HUCBMNCs, pretreated for $24 \mathrm{~h}$, were considered as 0-day CD3+CD56+ NKT-like cells. These were transferred to T75 culture flasks at a density of $5 \times 10^{6}$ cells $/ \mathrm{mL}$, and divided into four groups, each incubated with a different polysaccharide, as detailed in Table 1. Four injectable polysaccharides, including LNT, BCG-PSN, GPS, and SFPS, were obtained from the Cancer Hospital of The University of Chinese Academy of Sciences (Hangzhou, China). The treatment dose was according to the manufacturers' instructions. Control cells were left untreated.

From day 0 onwards, half of the medium of the co-cultures was replaced every other day with fresh complete medium containing $1000 \mathrm{U} / \mathrm{mL}$ of IL-2 and the polysaccharides at their respective concentration. The medium of the control cocultures was replaced with fresh medium containing $1000 \mathrm{U} / \mathrm{mL}$ of IL-2 only. The different cell populations were quantified in order to monitor the proliferation rate over 4 days. The cell populations were quantified using haemocytometer under a Nikon SMZ1500 microscope (Nikon Co., Ltd., Japan), and their mean numbers were calculated based on three replicates. The HUCBMNCs were co-cultured with various injectable polysaccharides for approximately 7 days before being harvested.

\subsection{Phenotypic and Functional Analysis of Polysaccharide-treated NKT-like Cells}

The phenotype of the CD3+CD56+ NKT-like cells was analyzed by flow cytometry. After 7 days in culture, $10^{7}$ CD3+CD56+ NKT-like cells were harvested and washed twice with phosphate buffered saline (PBS, pH 7.2-7.4, cell culture grade, Shenggong Ltd., China), and then stained for 30 minutes at room temperature with FITCconjugated anti-CD3 (Biolegend, USA) and PerCP-conjugated anti-CD56 (Biolegend, USA) antibodies. Next, the cells were washed three times with PBS and analyzed with a flow cytometer (Becton Dickinson FACS, USA). The levels of IFN- $\gamma$ and TNF- $\alpha$ in NKT-like cells were quantified respectively with a Human IFN-y ELISA Kit and a Human TNF- $\alpha$ ELISA Kit (Beyotime, Ltd., China). The absorbance at 450 $\mathrm{nm}$ was measured on a Molecular Devices FlexStation 3 Reader. The data were recalculated by the representative standard curves of IFN- $y$ and TNF- $\alpha$ respectively.

The functions of NKT-like cells were investigated by co-incubation with cells from the lung cancer cell line A549, obtained from the China Center for Type Culture Collection (Wuhan, China) and maintained in RPMI-1640 (Sigma, Gillingham, UK) supplemented with $10 \%(\mathrm{v} / \mathrm{v})$ FCS (BioSera, Ringmer, UK) and $1 \%$ (v/v) L-glutamine (Sigma). The cell line was routinely tested for mycoplasma and remained free of infection during the whole time of the investigation. Because NKT-like cells share some features with NK cells, including the capacity to recognize and kill cells devoid of HLA class I molecules, their functions can be assessed in co-cultures with HLA class Inegative A549 cells. Polysaccharide-treated NKT-like cells were co-incubated for 12 hours with A549 cells at serial effector/target (E:T) ratios of $5: 1,10: 1,20: 1$ and 40:1. CCK8 assays were used to assess the antitumor activity of polysaccharide-treated NKT-like cells by measuring their cytotoxicity against cells from the A549 lung cancer cell line. The process followed the manufacturer's guidelines(Cell Counting Kit8, Beyotime, Ltd. China).

\subsection{Statistics Analysis}

Data were statistically analyzed using the DPS 7.05 statistical software [19]. The results are presented as mean \pm standard deviation (SD). Comparison between groups was done using one-way analysis of variance (ANOVA), and the 
difference between pairs of means was tested using the post hoc with least significantdifference test (LSD) test. Differences were considered significant for $p$ value $<0.05$.

\section{RESULTS}

\subsection{Effect of the Polysaccharides on the Population of CD3+CD56+ NKT-like Cells}

The effect of injectable polysaccharide preparations was tested by monitoring the response of treated HUCBMNCs-derived NKTlike (Fig. 1). Over 4 days of culture, the population of cells treated with BCG-PSN, GPS, and SFPS, as well as the population of the untreated control cells showed an increasing trend, contrary to the population of the LNTtreated cells. The population of the BCG-PSNtreated cells reached $(5.46 \pm 0.06) \times 10^{6}$ on the fourth day, corresponding to a 1.09-fold increase compared to the first day. The population of the GPS-treated cells reached $(5.44 \pm 0.06) \times 10^{6}$ on the fourth day, corresponding to a 1.09-fold increase compared to the first day. The population of the SFPS-treated cells reached $(5.22 \pm 0.18) \times 10^{6}$ on fourth day, representing a 1.04-fold increase compared to the first day. LNT-treatment had an inhibitory effect on the proliferation of NKT-like cells, whose population reached $(4.83 \pm 0.12) \times 10^{6}$ on the fourth day, representing a 0.97-fold decrease as compared to the first day. Additionally, on day 4, the populations of the BCG-PSN- and GPS-treated cells were not significantly different from that of the control cells. However, after 4 days, SFPSand LNT-treated cultures had significantly decreased cell numbers compared to the control culture, with $p<0.05$ and $p<0.01$, respectively.

\subsection{Effect of the Polysaccharides on Cytokine Production by NKT-like Cells}

To determine the effect of the different polysaccharides on cytokine production by NKTlike cells, staining for TNF- $\alpha$ and IFN- $\gamma$ was performed using corresponding kits. Treatment with injectable polysaccharides resulted in a significant increase of TNF- $\alpha$ and IFN- $y$ concentration $(p<0.05)$ (Table 2). That is, IFN-Y production was the highest in the cells treated with GPS (1214.97 $\pm 20.75 \mathrm{pg} / \mathrm{mL})$. BCG-PSN and SFPS treatments resulted in similar concentration of IFN- $\gamma(p>0.05)$, respectively of $1144.03 \pm 8.65 \mathrm{pg} / \mathrm{mL}$ and $1032.03 \pm 39.46$ $\mathrm{pg} / \mathrm{mL}$. There was no significant difference in IFN- $\gamma$ concentration $(p>0.05)$ after LNT treatment $(794.12 \pm 47.52 \mathrm{pg} / \mathrm{mL})$ compared with control $(884.67 \pm 130.44 \mathrm{pg} / \mathrm{mL})$. Additionally, all four polysaccharides, in decreasing order GPS, SFPS, BCG-PSN, and LNT, induced significantly higher concentrations of TNF- $\alpha$ compared to control, with respectively: $274.87 \pm 7.27 \mathrm{pg} / \mathrm{mL}$; $262.93 \pm 14.22 \mathrm{pg} / \mathrm{mL} ; 249.43 \pm 14.75 \mathrm{pg} / \mathrm{mL}$; $214.52 \pm 7.43 \mathrm{pg} / \mathrm{mL}$; and $130.65 \pm 7.44 \mathrm{pg} / \mathrm{mL}$. These results indicate that HUCBMNCs-derived NKT-like cells can discriminate the different polysaccharides according to their ability to induce variable levels of cytokine production.

\subsection{Effect of the Polysaccharides on the Proportion of CD3+CD56+ NKT-like Cells}

The immunophenotype of the NKT cells treated with different polysaccharides for 7 days was examined by flow cytometry. The percentage of CD3+CD56+ cells, representing the main NKT effector cells, was determined after treatment with the different polysaccharides. The data showed that three of the tested polysaccharides, i.e., BCG-PSN, GPS, and SFPS, induced significantly higher proportion of CD3+CD56+ NKT-like cells, with respectively $27.61 \%$, $43.12 \%$, and $31.91 \%$, compared with control $(23.1 \%)(p<0.05)$ (Fig. 2). In contrast, the proportion of these cells was decreased to $19.47 \%$ upon LNT treatment. Furthermore, the proportion of CD56+ cells in the cultures treated with either of the four polysaccharides were higher than in untreated control. In decreasing order, these proportions were: $44.27 \%$ with GPS, $32.22 \%$ with SFPS, $28.07 \%$ with BCG-PSN, $27.14 \%$ with LNT, and $23.53 \%$ in control. Due to a lower proportion of CD3+ cells in the LNTtreated culture $(91.82 \%)$, the proportion of CD3+CD56+ NKT-like cells appeared to be the lowest compared to the proportions obtained in the other cultures, including the untreated control.

\subsection{Effect of the Polysaccharides on the Cytolytic Activity of the NKT-like Cells against Tumor Cells In vitro}

The antitumor activity of polysaccharide-treated NKT-like cells was assessed by measuring their cytotoxicity against cells from the A549 lung cancer cell line using the CCK8 assay. The cytotoxic activity induced by the different polysaccharides was compared with different effector to target (E:T) ratios. As shown in Fig. 3, 
polysaccharides-treated NKT cells killed A549 cells to varying degrees. Furthermore, the polysaccharide treatments increased the cytotoxicity of the NKT-like cells toward the tumor cells. GPS-treated NKT-like cells had the highest cytolytic activity, which reached $96.4 \%$, and had a significantly superior inhibitory effect on A549 cells, in all four $E / T$ ratios tested, ranging from 5:1 to 40:1, compared with control NKT-like cells $(P<0.01)$. SFPS-treated NKT-like cells also showed significant cytolytic activity at various $\mathrm{E} / \mathrm{T}$ ratios $(P<0.05)$. BCG-PSN-treated NKT-like cells had significantly higher cytolytic activity from the 10:1 ratio onwards $(P<0.05)$, with the degree of significance of the treatment-induced enhancement increasing with the $\mathrm{E} / \mathrm{T}$ ratio. However, at 5:1, LNT-treated NKT cells showed the lowest inhibitory effect, decreasing even below that of the control NKT-like cells $(15.8 \% \pm$ $27.2 \%$ vs $21.2 \% \pm 12.9 \%)$. Taken together, these results suggest that GPS treatment has the highest ability to increase the cytolytic activity of NKT-like cells against tumors.

Table 1. Polysaccharide injections list used in this study

\begin{tabular}{llll}
\hline Type & Manufacturer & Specification & Lot No \\
\hline Lentinan (LNT) & A & $1 \mathrm{mg} \mathrm{in} 2 \mathrm{~mL}$ & $\mathrm{H} 20030131$ \\
BCG polysaccharide nucleic acid (BCG- & B & $0.35 \mathrm{mg}$ in $1 \mathrm{~mL}$ & S33020001 \\
PSN) & C & $12 \mathrm{mg} \mathrm{in} 4 \mathrm{~mL}$ & $\mathrm{H} 14023513$ \\
Ginseng polysaccharide (GPS) & D & $10 \mathrm{mg} \mathrm{in} 10 \mathrm{~mL}$ & $\mathrm{Z} 51020664$ \\
Shengfu polysaccharide (SFPS) & &
\end{tabular}

Table 2. Comparison of the ability of the different polysaccharides to induce cytokine production by NKT-like cells using ANOVA

\begin{tabular}{lll}
\hline Treatment & IFN- $\mathbf{~} \mathbf{~ g} / \mathbf{m L}$ (Mean \pm SD) & TNF- $\mathbf{\alpha} \mathbf{~} \mathbf{g} / \mathbf{m L}$ (Mean \pm SD) \\
\hline Control & $884.67 \pm 130.44 \mathrm{c}$ & $130.65 \pm 7.44 \mathrm{~d}$ \\
LNT & $794.12 \pm 47.52 \mathrm{c}$ & $214.52 \pm 7.43 \mathrm{c}$ \\
BCG-PSN & $1144.03 \pm 8.65 \mathrm{ab}$ & $249.43 \pm 14.75 \mathrm{~b}$ \\
GPS & $1214.97 \pm 20.75 \mathrm{a}$ & $274.87 \pm 7.27 \mathrm{a}$ \\
SFPS & $1032.03 \pm 39.46 \mathrm{~b}$ & $262.93 \pm 14.22 \mathrm{ab}$ \\
\hline
\end{tabular}

Note: IFN- $y$ and TNF- $\alpha$ production in CD3+CD56+ NKT-like cells following different polysaccharides treatment.

The data are representative of two independent experiments with similar results and are expressed as the mean $\pm S D$ ( $n=3$ for each experiment). Means followed with different lowercase letters in each column differed significantly (Tukey's HSDs, $p<0.05$ ).

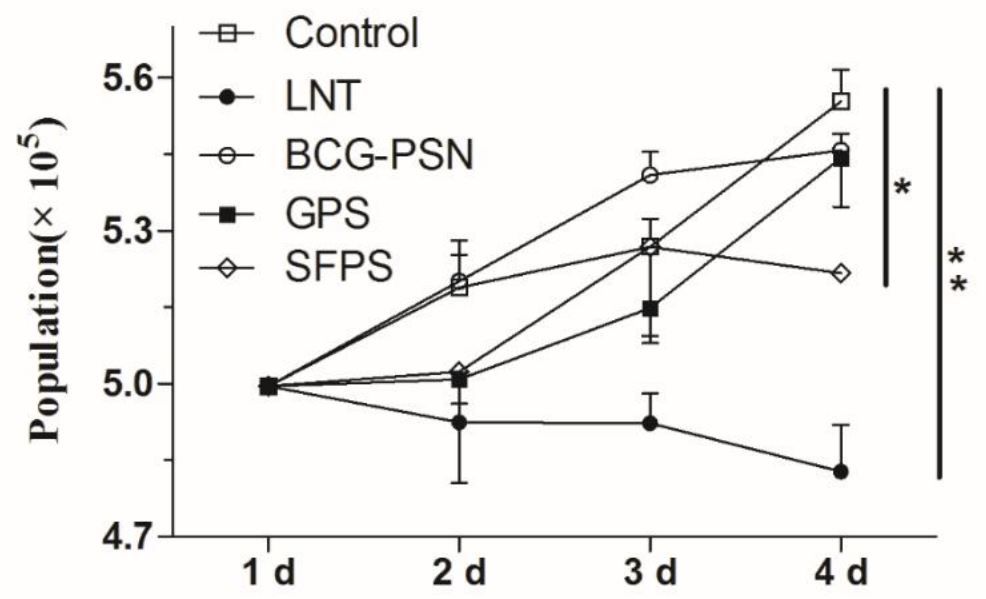

Fig. 1. Effect of different polysaccharides on the number of NKT-like cells $\left({ }^{*} p<0.05,{ }^{* *} p<0.01\right.$ mean significant levels between treatment group and the control group) 

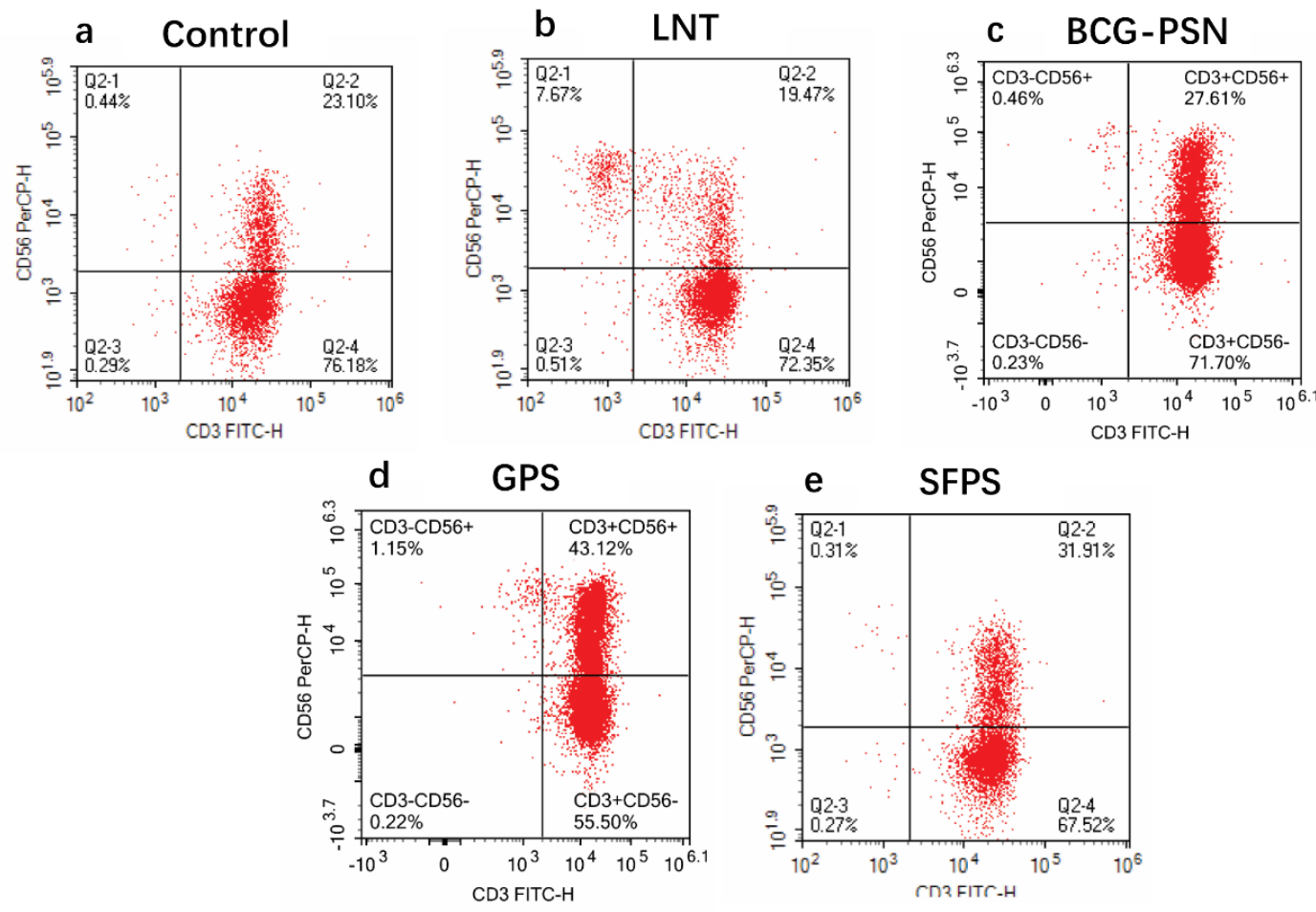

Fig. 2. Representative dot-plot showing HUCBMNCs-derived NKT-like cells stained with FITCconjugated anti-CD3 monoclonal antibodies and PerCP-conjugated anti-CD56 monoclonal antibodies

Percentage of $C D 3+C D 56+N K T$ cells (number in upper-right quadrants of each dot plot) after 7 days in culture (a) untreated (control), (b) with LNT, (c) with BCG-PSN, (d) with GPS, and (e) with SFPS. The proportion of $C D 3+C D 56+N K T$ cells after treatment with three different polysaccharides (c: $27.61 \%$, d: $43.12 \%$, e: $31.91 \%$ ) was significantly higher than in untreated control (a:23.1\%). However, the proportion of these cells decreased upon LNT treatment (b: 19.47\%)

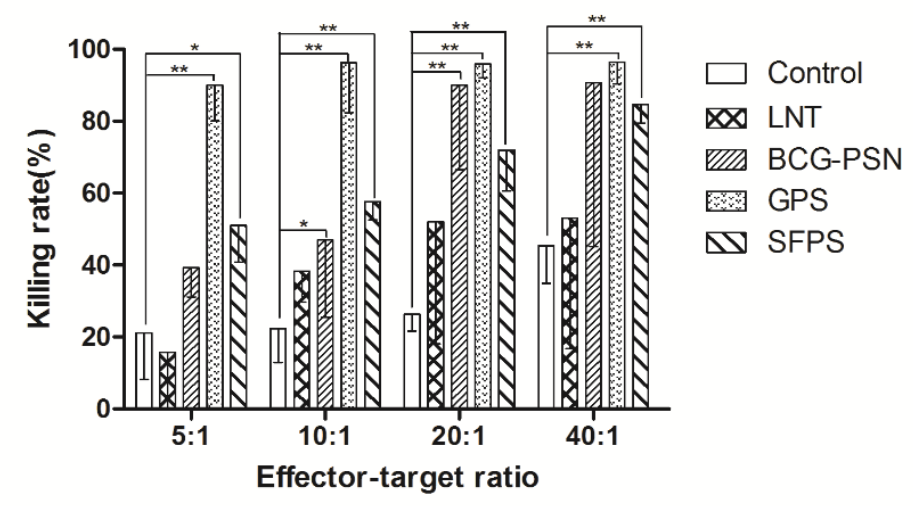

Fig. 3. Polysaccharides enhance the cytolytic activity of NKT-like cells against tumor cells from the A549 line in vitro

NKT-like cells were harvested and analyzed by cytotoxicity assay. Antitumor effects of NKT-like cells treated with different polysaccharides, including LNT, BCG-PSN, GPS and SFPS, or untreated (Control) on cells from the A549 tumor line. ${ }^{*} p<0.05,{ }^{* *} p<0.01$ versus the control group 


\section{DISCUSSION}

The use of polysaccharides to induce immunity and regulate immune cell responses has broad research prospects $[20,21]$. Thus, to develop a novel method to evaluate the potential of injectable polysaccharides represents an absolute requirement toward further testing in clinical trials. Cord blood-derived CD3+CD56+ NKT-like cells were chosen as a readout population because of one of their specific characteristics, which is that they are absent from the cord blood, but increase gradually in absolute number in older individuals due to repeated antigen exposure [18].

The newly developed evaluation system, relying on CD3+CD56+ NKT-like cells, was stable for the four injectable polysaccharides assessed. The number of CD3+CD56+ NKT-like cells after treatment with LNT BCG-PSN, GPS, or SFPS was lower than in untreated cultures (Fig. 1). The reason for this decrease might be that polysaccharides exert a significant clinical efficacy through inducing cancer cell apoptosis, inhibiting metastatic cell proliferation [2], affect the NKT cells as well. Among the tested polysaccharides, GPS and BCG-PSN treatments resulted in increased proliferation of the NKT-like cells similarly to untreated conditions, but to a lower extent. This effect of GPS was consistent with those observed in other reports, indicating that polysaccharides can promote the proliferation of immune cells [22], but have multiple immunomodulatory and inhibitory effects on A549 cell growth, as well as pro-apoptotic effect [9]. Biomacromolecules constitutive of BCG-PSN, including polysaccharides, nucleic acids, proteins, and lipids, participate in multiple biological activities, such as cell-to-cell communication and immune regulation [8]. In the meantime, the active ingredients of SFPS are mainly ginsenoside, aconitine, and ginseng polysaccharide. Pharmacological studies demonstrated that aconitine exerts an inhibitory effect on proliferation, invasion, and metastasis of the human adenocarcinoma A549 cell lines through decreasing MMP-2 and MMP-9 activity [23]. Therefore, the proliferation of SFPS-treated NKT-like cells was significantly lower than that of the untreated control cells. The particular decreasing effect of LNT on NKT-like cell proliferation was consistent with other reports showing that Lentinan does not accelerate antibody formation, nor does it cause an increase of blood lymphocyte count, accelerate allograft rejection, or influence delayed hypersensitivity reactions [7].

Polysaccharide treatment not only activates macrophages, lymphocytes, lymphoid factorsactivated killer cells, toxic $\mathrm{T}$ cells, and dendritic cells but also promotes the production of cytokines, activates the complement system, and accelerates the production of antibodies [24,25]. After treatment with BCG-PSN, GPS, and SFPS (Table 2), NKT-like cells produced more IFN-Y and TNF-a, which are both important for the killing of tumor cells. This result was consistent with other studies on treatment of peripheral blood $[10,22,26]$. In contrast, LNT treatment induced only little IFN-y production by NKT-like cells, which was not significantly different from the level produced by the untreated control (Table 2). The reason for this result might be that fungal polysaccharide LNT injections were rather considered for their immunoregulatory properties in clinical trial [27].

The cytolytic activity of NKT-like cells induced by GPS, BCG, PSN, and SFPS were accompanied with increasing proliferation, cytokine production, and higher proportion of cells with a CD3+CD56+ phenotype. GPS induced the highest CD3+CD56+ proportion after 7 days of treatment, and the highest cytolytic activity against the A549 tumor cell line (Fig. 2, Fig. 3). This suggests that GPS might be the best injectable polysaccharides adjuvant to enhance the effectiveness of CD3+CD56+ NKT-like cells. Furthermore, among the cytokine-induced killer (CIK) cells used in transfusion as adoptive cellular immunotherapy for cancer, CD3+CD56+ NKT-like cells are known to be major effector cells and display the most potent cytolytic activity [28]. CIK cells can recognize and lyse tumor cell targets in a major histocompatibility complexunrestricted manner [28]. Moreover, they can also regulate immune functions by secreting various cytokines, such as tumor necrosis factor (TNF)- $\alpha$ and IFN- $\gamma$. Screening of polysaccharide adjuvants to enhance the effectiveness of adoptive cell immunotherapy such as CIK cellbased therapy would represent an attractive prospect.

Finally, this study was limited to the test of four common polysaccharides on CD3+CD56+ NKTlike cells proliferation and cytotoxicity. The mechanism of polysaccharide-induced NKT-like cell differentiation was not explored. Therefore, further polysaccharides and their respective mechanisms should be investigated in future. 


\section{CONCLUSION}

The cytolytic activity of NKT-like cells induced by GPS, BCG, PSN, and SFPS were accompanied with increasing proliferation, cytokine production, and higher proportion of cells with a CD3+CD56+ phenotype. GPS induced the highest CD3+CD56+ proportion after 7 days of treatment, and the highest cytolytic activity against the A549 tumor cell line. Conclusively, cord blood-derived CD3+CD56+ NKT-like cells may represent a powerful tool to screen and evaluate the immunoregulatory potential of biological or chemical compounds for clinical developments and applications.

\section{CONSENT AND ETHICAL APPROVAL}

As per international standard or university standard guideline participant consent and ethical approval has been collected and preserved by the authors.

\section{ACKNOWLEDGEMENTS}

The authors would like to thank the National Key Research and Development Plan (2018YFA0108403), the Zhejiang Natural Science Foundation (Y18C140002, LGN18C200026), the Natural Science Foundation of China (31461143030) and Outstanding Talents Training Program of "HuanYu" (01107180032) for having supported this project.

\section{COMPETING INTERESTS}

Authors have declared that no competing interests exist.

\section{REFERENCES}

1. Yin $M$, Zhang $Y$, Li $H$. Advances in research on immunoregulation of macrophages by plant polysaccharides. Front Immunol. 2019;10:1-9.

2. $Y u Y$, Shen $M$, Song $Q$, et al. Biological activities and pharmaceutical applications of polysaccharide from natural resources: a review. Carbohydr Polym. 2018;183:91101.

3. Shin MS, Hwang SH, Yoon TJ, et al. Polysaccharides from Ginseng leaves inhibit tumor metastasis via macrophage and NK cell activation. Int $\mathrm{J}$ Biol Macromol. 2017;103:1327-1333.
4. Zhang GW, Qin GF, Han B, et al. Efficacy of Zhuling polyporus polysaccharide with BCG to inhibit bladder carcinoma. Carbohydr Polym. 2015;118:30-35.

5. Wang M, Konishi T, Gao Y, et al. Antigastric ulcer activity of polysaccharide fraction isolated from mycelium culture of lion's mane medicinal mushroom, Hericium erinaceus (Higher Basidiomycetes). Int J Med Mushrooms. 2015;17:1055-1060.

6. Ferreira SS, Passos CP, Madureira P, et al. Structure-function relationships of immunostimulatory polysaccharides: A review. Carbohydr Polym. 2015;132:378396.

7. Ian RT, Yawei N. Carbohydrates, immune stimulating. Peter JD(Eds), Encyclopedia of Immunology (Second Edition), Elsevier. 1998;427-431.

8. Wei D, Chen T, Yan M, et al. Synthesis, characterization, antioxidant activity and neuroprotective effects of selenium polysaccharide from Radix hedysari. Carbohydr Polym. 2015;125:161-168

9. Zhao JP, Wang YY. Experimental study of the A549 cell apoptosis of human nonsmall cell lung cancer induced by Ginseng Polysaccharide in vitro. Chin J Integr Tradit \& Western Med. 2006; 6:95-97.

10. Cao $A$, He $H$, Jing $M$, et al. Shenfu injection adjunct with platinum-based chemotherapy for the treatment of advanced non-small-cell lung cancer: A meta-analysis and systematic review. Evid Based Complement Alternat Med 2017. 2017:1068751.

11. Jiang $Y$, Cui $X$, Cui $C$, et al. The function of CD3+CD56+ NKT-like cells in HIV-infected individuals. Biomed Res Int 2014. 2014;863625.

12. Kronenberg M. Toward an understanding of NKT cell biology: progress and paradoxes. Ann Rev Immunol. 2005;23: 877-900.

13. Loza MJ, Metelitsa LS, Perussia B. NKT and $T$ cells: Coordinate regulation of NKlike phenotype and cytokine production. Eur J Immunol. 2002;32:34533462.

14. Di Pietro $\mathrm{C}$, Falcone $\mathrm{M}$. The role of invariant NKT cells in organ-specific autoimmunity. Front Biosci. 2014;19:12401250.

15. Godfrey DI, Hammond KJ, Poulton LD, et al. NKT cells: Facts, functions and fallacies. Immunol Today. 2000;21:573583. 
16. Doherty DG, O'Farrelly C. Innate and adaptive lymphoid cells in the human liver. Immunol Rev. 2000;174:5-20.

17. Vitale $M$, Caruso $A$, Licenziati $S$, et al. Differential production of IFN-gamma, analyzed at the single-cell level, by specific subsets of human NK and $\mathrm{T}$ cells from healthy and $\mathrm{HIV}(+)$ subjects. Cytometry. 2000;39:189-194.

18. Giroux M, Denis F. CD1d-unrestricted human NKT cells release chemokines upon Fas engagement. Blood. 2005;105:703-710.

19. Tang QY, Feng MG. DPS data processing system for practical statistics, Science Press, Beijing;2002.

20. Wang $Y$, Wang BC, Zhou J, et al. Investigated on immune regulation of polysaccharide. J Chongqing Univ. 2004;27:104-107.

21. Hao X, Zhao MY, Dong ZM. Effect of Ganoderma lucidum polysaccharides and Lycium barbarum polysaccharide combined application of antitumor activity and immune function in tumor bearing mice. Tradit Chin Med Res. 2011;24:3335.Xie ZJ, Liu WQ, Fang $C L$, et al. Experimental studies on anti-tumor activity and effect of Ginseng polysaccharide on proliferative function of LAK cells. Chin $\mathrm{J}$ Clin Onocol \& Rehabilit. 2002;9:1-2.

22. Xu XF, Chen YH, Wang J, et al. Effect and mechanism of aconitum vaginatum on the proliferation, invasion and metastasis in human A549 lung carcinoma cells. J Chin Med Mater. 2010;33:1909-1912.

23. Sundberg-Kovamees M, Grunewald J, and Wahlstrom J. Immune cell activation and cytokine release after stimulation of whole blood with pneumococcal Cpolysaccharide and capsular polysaccharides. Int $\mathrm{J}$ Infect Dis. 2016;52:1-8.

24. Zhu N, Lv X, Wang Y, et al. Comparison of immunoregulatory effects of polysaccharides from three natural herbs and cellular uptake in dendritic cells. Int $\mathrm{J}$ Biol Macromol. 2016;93:940951.

25. Saunders B, Briscoe H, Britton W. T cellderived tumour necrosis factor is essential, but not sufficient, for protection against Mycobacterium tuberculosis infection. Clin Exp Immunol. 2004;137:279-287.

26. McCormack E, Skavland J, Mujic M, et al. Lentinan: hematopoietic, immunological, and efficacy studies in a syngeneic model of acute myeloid leukemia. Nutr Cancer. 2010;62:574-83.

27. Gao X, Mi Y, Guo N, et al. Cytokineinduced killer cells as pharmacological tools for cancer immunotherapy. Front Immunol. 2017;8:1-13.

28. Introna M, Correnti F. Innovative clinical perspectives for CIK cells in cancer patients. Int J Mol Sci. 2018;19:1-14.

(c) 2021 Lu et al.; This is an Open Access article distributed under the terms of the Creative Commons Attribution License (http://creativecommons.org/licenses/by/4.0), which permits unrestricted use, distribution, and reproduction in any medium, provided the original work is properly cited.

Peer-review history:

The peer review history for this paper can be accessed here: http://www.sdiarticle4.com/review-history/67907 